\title{
An observational study of intubation success rates and rescue airway techniques among 7256 pre-hospital physician intubations of trauma patients
}

\author{
Kate Crewdson ${ }^{*}$, David J Lockey \\ From London Trauma Conference 2013 \\ London, UK. 10-13 December 2013
}

\section{Background}

Effective airway management is a priority in early trauma management. Data on physician pre-hospital intubation is limited, despite its worldwide practice [1]. This study was conducted to establish intubation success rates in a physician-led system and examine the frequency and management of failed intubation in pre-hospital trauma patients. Failed intubation rates of anaesthetists and nonanaesthetists were also reviewed.

\section{Method}

A retrospective database review was conducted to identify trauma patients undergoing pre-hospital advanced airway management between September 1991 and December 2012. Intubation success rates and success rate of individuals and by speciality were recorded. The use and success of rescue techniques were also established.

\section{Results}

The doctor- paramedic team attended 28,939 trauma patients; $7256(25.1 \%)$ required advanced airway management. Forty-six patients $(0.6 \%)$ had immediate surgical airway performed without any attempted intubation. Of the remaining 7210 patients, intubation was successful in 7158 (99.3\%). Rescue surgical airways were performed in 42 patients, seven had successful insertion of supraglottic devices, two patients had supraglottic device insertion and a surgical airway. One patient was allowed to breathe spontaneously with bag-valve-mask support during transfer. All rescue techniques were successful.

London's Air Ambulance, Royal London Hospital, London, United Kingdom
Non-anaesthetists performed 4394 intubations and failed to intubate in 41 cases $(0.9 \%)$; anaesthetists performed 2587 intubations and failed in $11(0.4 \%)(\mathrm{p}=$ $0.02)$. Forty-one of 186 doctors $(22 \%)$ had at least one failed intubation.

\section{Conclusion}

This study reports the largest series of physician pre-hospital intubations. The reported success rate $(99.3 \%)$ is consistent with other published series (median 99.1\%) [1]. All rescue airway techniques were successful. Non-anaesthetists were twice as likely to have to perform a rescue airway intervention than anaesthetists and this difference was statistically significant. Surgical airways were performed using a standard surgical technique. The rate of surgical airway reported here $(0.7 \%)$ is lower than in many other physicianled series (median $3.1 \%$, range $0.1 \%-7.7 \%$ ) [2,3].

\section{Published: 7 July 2014}

\section{References}

1. Lossius HM, Røislien J, Lockey DJ: Patient safety in pre-hospital emergency tracheal intubation: a comprehensive meta-analysis of the intubation success rates of EMS providers. Crit Care 2012, 16:R24.

2. Timmermann A, Eich C, Russo SG, Natge U, Bräuer A, Rosenblatt WH, Braun U: Prehospital airway management: a prospective evaluation of anaesthesia trained emergency physicians. Resuscitation 2006, 70:179-185.

3. Xeropotamos NS, Coats TJ, Wilson AW: Prehospital surgical airway management: 1 year's experience from the Helicopter Emergency Medical Service. Injury 2013, 24:222-224.

doi:10.1186/1757-7241-22-S1-P13

Cite this article as: Crewdson and Lockey: An observational study of intubation success rates and rescue airway techniques among 7256 prehospital physician intubations of trauma patients. Scandinavian Journal of Trauma, Resuscitation and Emergency Medicine 2014 22(Suppl 1):P13. 